\title{
Serum levels of sex hormone-binding globulin (SHBG) are not associated with lower levels of non-SHBG-bound testosterone in male newborns and healthy adult men
}

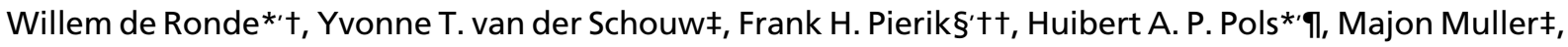 \\ Diederick E. Grobbeeł, Louis J. G. Goorent, Rob F. A. Webertt and Frank H. de Jong* \\ Departments of ${ }^{\star}$ Internal Medicine, Public Health, EEidemiology and Biostatistics and $\dagger \dagger$ Andrology, Erasmus Medical Center, \\ Rotterdam, $\dagger$ Department of Endocrinology, Vrije Universiteit Medical Center, Amsterdam and $\ddagger J u l i u s$ Center for Health Sciences \\ and Primary Care, University Medical Center, Utrecht, the Netherlands
}

\section{Summary}

Objective It is generally accepted that SHBG decreases the bioavailability and activity of testosterone $(\mathrm{T})$. In in vitro experiments increased levels of SHBG will be associated with decreased levels of non-SHBG bound testosterone (non-SHBG-T). However, in vivo SHBG can alter both production and clearance rates and thus plasma levels of $\mathrm{T}$.

Design and patients In order to study the effect of SHBG on the levels of non-SHBG-T in vivo in the presence of an active hypothalamo-pituitary-gonadal (HPG) axis we conducted a cross sectional study in 400 healthy adult men with an age range of $40-$ 80 years and in 106 newborn boys.

Measurements In both groups, regression coefficients $(\beta)$ and partial correlation coefficients ( $r$ ) were calculated for the relationship between SHBG and T or non-SHBG-T. Adult men were divided into age groups per decade ( $40-50$ years, $51-60$ years, $61-70$ years and $71-80$ years) to study possible differences in the impact of SHBG on the level of non-SHBG-T throughout ageing.

Results Higher levels of SHBG were associated with higher levels of total testosterone in neonates $(\beta=0.02 \pm 0.004, r=0.44$, $P<0.001)$ but not with non-SHBG-T $(\beta=-0.001 \pm 0.001, r=0.05$, $P=0.52)$. In adult men there was a significant age related increase in levels of SHBG and an age-related decrease of both total and non-SHBG-T. Higher SHBG was strongly associated with higher total testosterone in all age groups $(\beta=0 \cdot 26,0 \cdot 26,0.26$ and 0.23 for 40 50 years, $51-60$ years, $61-70$ years and $71-80$ years, respectively, $P<0.001$ for all age groups). Higher SHBG was not or only slightly associated with higher non-SHBG-T $\beta=0.02(P=0.32), \beta=0.04$ $(P=0.03), \beta=0.04 \quad(P=0.02)$ and $\beta=0.02 \quad(P=0.16)$ for $40-$ 50 years, $51-60$ years, $61-70$ years and $71-80$ years, respectively. Conclusions In contrast to general belief, SHBG levels barely influence levels of non-SHBG-bound testosterone both in male newborns

Correspondence: Frank H. de Jong Ph.D., Endocrine Laboratory, room Ee 516, Department of Internal Medicine, Erasmus MC, PO Box 1738, 3000 DR Rotterdam, the Netherlands. E-mail: f.h.dejong@erasmusmc.nl and healthy adult men: the influence, if any, is positive. Consequently the age related increase of SHBG does not account for the age related decline in non-SHBG-T in healthy adult men.

(Received 3 August 2004; returned for revision 20 October 2004; finally revised 12 January 2005; accepted 7 February 2005)

\section{Introduction}

Sex-hormone binding globulin (SHBG), corticosteroid binding globulin (CBG) and albumin are important steroid hormone binding proteins in human plasma. In normal adult men approximately $44 \%$ of the circulating testosterone is specifically bound to SHBG, $50 \%$ is nonspecifically bound to albumin and $3.5 \%$ is bound to CBG implicating that only $2-3 \%$ is unbound or free. ${ }^{1}$ By binding testosterone, SHBG decreases the metabolic clearance rate of testosterone $\mathrm{e}^{2,3}$ and decreases peripheral conversion of testosterone to androstenedione. ${ }^{2}$ To exert its genomic action, testosterone must bind to an intracellular androgen receptor. SHBG withholds bound hormone to diffuse from the bloodstream as free hormone to this receptor. There is some controversy whether or not albumin-bound testosterone can dissociate freely from this carrier protein and enter tissues; ${ }^{4,5}$ however, non-SHBG bound testosterone (nonSHBG-T $=$ free + albumin bound testosterone) and non-SHBGnonalbumin bound testosterone (= free testosterone) are extremely well-correlated ${ }^{6}$ and interchangeable in most cases.

The levels of SHBG vary widely between healthy men and depend on factors such as diet, ${ }^{7}$ body mass index (BMI), insulin levels and age. ${ }^{8}$ On the basis of simple mathematics a higher level of SHBG is expected to result in a lower level of non-SHBG-T. However, when the non-SHBG-T is considered bioactive, variations in SHBG concentrations will cause a variable secretion of LHRH by the hypothalamus and LH secretion by the pituitary. Low levels of non-SHBG-T will then lead to increased LH and testicular testosterone production via a decreased negative feedback signal on the hypothalamus and pituitary. Estimation of the effect of SHBG on the levels of non-SHBG-T in vivo by simply applying the laws of mass 
action might thus be inappropriate in men with a functional hypothalamo-pituitary-gonadal (HPG) axis.

In healthy males, two phases of life are characterized by high gonadal axis activity; the neonatal period and the period during and after puberty. ${ }^{9}$ In the first 6 months of life, levels of both LH and T are high, reaching levels in the low adult range. ${ }^{10}$ At the age of 6 months, both LH and T levels have decreased to very low levels only to increase at the start of puberty. ${ }^{11}$ In adult men, T levels remain high throughout life although ageing is associated with a slight decrease of the levels of total serum testosterone and an increase of the levels of SHBG. ${ }^{12}$

To study the effect of SHBG on the levels of non-SHBG-T in vivo we conducted a cross-sectional study in 400 healthy adult men with an age range of 40-80 years and in 106 newborn boys.

\section{Subjects and methods}

\section{Neonates}

Serum samples were collected from 112 boys with ages between 1 and 6 months who served as controls in a case-control study on cryptorchism and hypospadias. Controls had been randomly selected from the population of boys that visited Child Healthcare Centers (CHCs). In the Netherlands, $\mathrm{CHCs}$ are notified of live births within 2 days after registration in the municipal birth register. $\mathrm{CHCs}$ invite all parents to participate free of charge in the national preventive child health care programme, including growth monitoring and vaccination, in which $95 \%$ of all parents participate with their child. This study was approved by the ethical committee of the Erasmus Medical Center, Rotterdam. Six subjects were excluded for analysis because of missing data on SHBG (five patients) or age (one patient). Eventually 106 subjects were available for analysis.

\section{Laboratory measurements in neonates}

Testosterone levels were estimated using a nonextraction coated tube radioimmunoassay (Coat-a-Count, Diagnostic Products Corporation, Los Angeles, CA, USA). The lower limit of detection of the assay was $0 \cdot 1 \mathrm{nmol} / \mathrm{l}$. Intra- and interassay coefficients of variation were below $9 \%$ for the concentration range measured in these samples. SHBG concentrations were measured using a chemoluminescencebased immunometric method (Immulite 2000) from the same supplier. The lower limit of detection was $5 \mathrm{nmol} / \mathrm{l}$. Variations for this method were below $7 \%$. Non-SHBG bound T was calculated using the method described by Sodergard et al. ${ }^{13}$ using a fixed albumin level of $40 \mathrm{~g} / \mathrm{l}$. The formulas for these calculations have been described earlier. ${ }^{14}$

\section{Adult subjects}

The study is a cross-sectional, single-centre study in 400 independently living men aged $40-80$ years. The study was originally designed to study the relationships between endogenous sex hormones and risk factors or manifestations of chronic diseases. The subjects were recruited in two ways. First, by asking female participants of other studies conducted by the department whether they knew a possibly interested male volunteer. Invitation letters were sent to 770 female participants. Eventually, 240 men volunteered for participation. Second, names and addresses of a randomly selected male population aged 40-80 years were drawn from the municipal register of Utrecht, a large-sized town in the central part of the Netherlands. 1230 invitation letters were send. From this group, 390 men volunteered for participation.

The subjects who did not live independently or were not physically or mentally able to visit the study centre independently $(n=16)$ were excluded. No additional health related eligibility criteria were used. Of the remaining 614 men, 400 men were randomly selected to participate. One subject was excluded from analysis because of clear hypogonadism (total testosterone $=0 \cdot 24 \mathrm{nmol} / \mathrm{l}$ ). To obtain equal numbers in each age-decade we sampled 100 men in each decade of age. Data collection took place between March 2001 and April 2002.

All participants gave written informed consent before enrolment and the institutional review board of the Utrecht University Medical Centre approved the study. Height and weight were measured in standing position without shoes. BMI was calculated as the weight in kilograms divided by the square of height in metres. Details on lifestyle and health of the subjects have been published earlier. ${ }^{15}$

\section{Laboratory measurements in adult subjects}

Fasting blood samples were obtained by venepuncture. Cell free serum was immediately stored at $-20^{\circ} \mathrm{C}$. T was measured after diethylether extraction using an in house radioimmunoassay employing a polyclonal antiT-antiserum (AZG 3290 Dr JJ Pratt, Groningen, the Netherlands). The lower limit of detection of the assay was $0 \cdot 24 \mathrm{nmol} / 1$ and interassay variation was $6 \cdot 0,5 \cdot 4$, and $8 \cdot 6 \%$ at $2 \cdot 1$, 5.6 and $23 \mathrm{nmol} / \mathrm{l}$, respectively. Results using this assay were comparable to those obtained with the assay used for estimation of $\mathrm{T}$ in neonates $(r=0 \cdot 91, n=37$, slope of regression line 1.03, intercept on $y$-axis $0.37 \mathrm{nmol} / \mathrm{l})$. SHBG was measured as described for the measurement in neonates. Non-SHBG bound $\mathrm{T}$ was calculated as described above.

\section{Data analysis}

All variables, except for age in neonates, were normally distributed. In neonates, age was logarithmically transformed to obtain normality. Associations between SHBG, total and non-SHBG-bound testosterone were assessed and tested for significance using linear regression analysis with $\mathrm{SHBG}$ as the independent variable and expressed as regression coefficient $(\beta)$ or (partial) correlation coefficient ( $r$ ). The linear regression coefficient $\beta$ indicates the change of the dependent variable for every $1 \mathrm{nmol} / \mathrm{l}$ change in SHBG. With every regression analysis, residuals were checked for normality. In both groups, analyses were repeated with adjustments for age (days) in neonates, and adjustments for age (years) and BMI in adult men. Adjustments for age and BMI were made by adding these parameters as independent variables to the regression and correlation model. Adjustments were made because both age and BMI were shown to be associated with levels of SHBG and T in men. ${ }^{10,15}$ Adult men were studied as a group and after division into age groups per decade (40-50 years, 51-60 years, 61-70 years and 71-80 years). Effect 

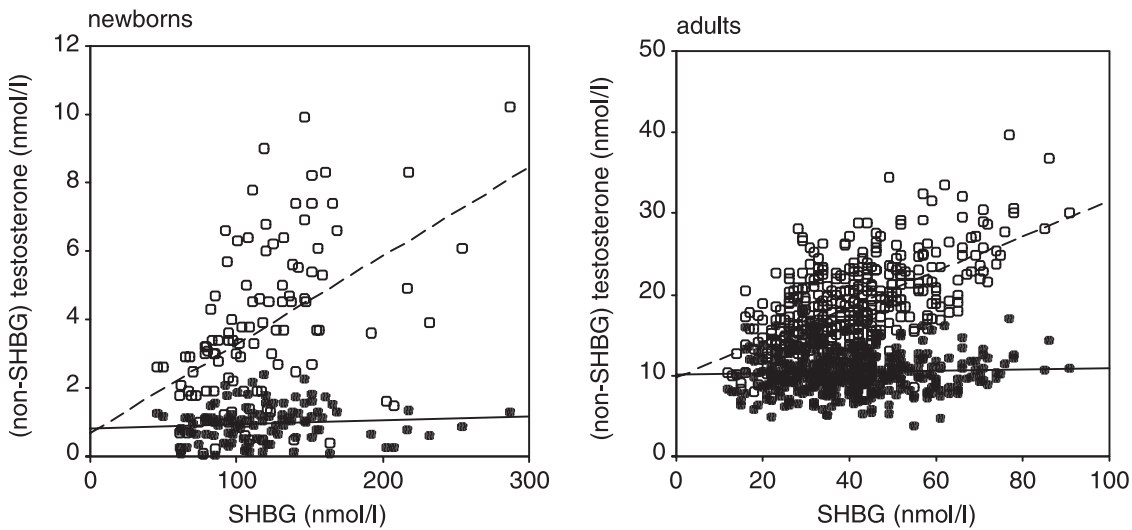

Fig. 1 SHBG vs. total testosterone (open symbols) and non-SHBG-T (closed symbols) in newborn boys and adult men.

Table 1. Levels of (non-SHBG-bound) testosterone and SHBG in newborns and adult men (mean $\pm \mathrm{SD})$

\begin{tabular}{lcc}
\hline & Newborns & Adult men \\
\hline$n$ & 106 & 399 \\
Age (days/years) & $79 \cdot 1 \pm 27 \cdot 3$ & $60 \cdot 2 \pm 11 \cdot 3$ \\
SHBG (nmol/l) & $118 \pm 43$ & $40 \cdot 6 \pm 14 \cdot 5$ \\
Testosterone (nmol/l) & $3 \cdot 73 \pm 2 \cdot 30$ & $18 \cdot 6 \pm 5 \cdot 26$ \\
Non-SHBG-T (nmol/l) & $0 \cdot 97 \pm 0 \cdot 53$ & $10 \cdot 4 \pm 2 \cdot 62$ \\
\hline
\end{tabular}

modification by age was tested using a linear regression model in which BMI, age group, SHBG and an interaction term (SHBG ${ }^{\star}$ age group) were used as independent variables and total testosterone or non-SHBG-T as dependent variables. Analyses were done using SPSS $11 \cdot 0$ software (SPSS, Chicago, IL, USA).

\section{Results}

The characteristics of newborns and adult men are presented in Table 1. In newborns levels of SHBG were much higher and levels of both total and unbound testosterone were much lower compared to adults. Higher levels of SHBG were significantly associated with higher levels of total testosterone in neonates but levels of SHBG were not significantly associated with levels of non-SHBG-T (Unadjusted, for T: $\beta=0 \cdot 03 \pm 0 \cdot 005, r=0 \cdot 49, P<0 \cdot 001$; for non-SHBG-T: $\beta=0 \cdot 001 \pm 0 \cdot 001, r=0 \cdot 09, P=0 \cdot 35$. After adjustment for age (days), for T: $\beta=0.02 \pm 0.004, \quad r=0.45, \quad P<0.001$; for non-SHBG-T: $\beta=0.001 \pm 0 \cdot 001, r=0 \cdot 06, P=0 \cdot 52)$. The relations between SHBG and total or unbound testosterone in neonates are depicted in Fig. 1.
A similar pattern was seen in adult men (Fig. 1; Unadjusted, for T: $\beta=0.22 \pm 0.02, r=0.59, P<0.001$; for non-SHBG-T: $\beta=0.01 \pm$ $0 \cdot 01, r=0.05, P=0.35)$. After adjustment for age and BMI the slightly positive association between SHBG and non-SHBG-T was statistically significant (for T: $\beta=0.25 \pm 0.01, r=0.68, P<0.001$; for non-SHBG-T: $\beta=0 \cdot 03 \pm 0 \cdot 01, r=0 \cdot 15, P=0 \cdot 01)$.

The characteristics of adult men after division in age groups are presented in Table 2. There was a significant age-related increase in levels of SHBG and a significant age-related decrease of both total and non-SHBG-T $(P<0.001$ for all variables). Relationships between SHBG and total or unbound testosterone after adjustments for age and BMI are presented in Table 3 and Fig. 2. Higher levels of SHBG were strongly associated with higher levels of total testosterone in all age groups. Levels of SHBG were not or marginally positively associated with levels of non-SHBG-T. There was no modifying effect of age on the associations between SHBG and total testosterone $(P$ for interaction $=0 \cdot 36)$ or non-SHBG-T $(P$ for interaction $=0 \cdot 94)$.

\section{Discussion}

This study in a large number of neonates and men across a wide age range confirms that levels of SHBG can vary widely among individuals. ${ }^{8}$ Our results show that there is a strong and positive relation between SHBG and total testosterone levels in both newborn boys and adult men. Moreover there is no, or only a weak positive association between SHBG levels and levels of circulating non-SHBG-T. These associations are completely different compared to the relationships anticipated on the basis of mathematical models. ${ }^{1}$ In these models, SHBG does not have an effect on the level of total testosterone. However, in vivo SHBG can have an effect on clearance and

\begin{tabular}{lllll}
\hline Age group (years) & $40-50$ & $51-60$ & $61-70$ & $71-80$ \\
\hline$n$ & 100 & 100 & 100 & 99 \\
Age (years) & $45 \cdot 0 \pm 3 \cdot 12$ & $56 \cdot 2 \pm 2 \cdot 89$ & $65 \cdot 4 \pm 2 \cdot 77$ & $74 \cdot 4 \pm 2 \cdot 69$ \\
SHBG (nmol/l)*** & $34 \cdot 7 \pm 13 \cdot 7$ & $38 \cdot 0 \pm 13 \cdot 3$ & $43 \cdot 6 \pm 15 \cdot 0$ & $46 \cdot 1 \pm 13 \cdot 2$ \\
Testosterone $(\mathrm{nmol} / \mathrm{l})^{* * *}$ & $20 \cdot 2 \pm 5 \cdot 59$ & $18 \cdot 5 \pm 5 \cdot 22$ & $17 \cdot 9 \pm 5 \cdot 53$ & $17 \cdot 8 \pm 4 \cdot 32$ \\
Non-SHBG-T (nmol/1)*** & $12 \cdot 2 \pm 2 \cdot 75$ & $10 \cdot 6 \pm 2 \cdot 37$ & $9 \cdot 57 \pm 2 \cdot 42$ & $9 \cdot 29 \pm 1 \cdot 79$ \\
\hline
\end{tabular}

Table 2. Levels of (non-SHBG-bound) testosterone and SHBG in adult men by age decade $($ mean $\pm \mathrm{SD})$

${ }^{* * *} P<0.001$ for differences between age groups. 
Table 3. Multiple linear regression coefficients $(\beta)$ and partial correlation coefficients ( $r$ ) for the relation between SHBG, testosterone and non-SHBG-bound testosterone in adult men for the total population $(n=399)$ and by age decade

\begin{tabular}{|c|c|c|c|c|c|c|c|c|c|c|}
\hline & \multicolumn{2}{|c|}{ Total population } & \multicolumn{8}{|c|}{ Age group (years) } \\
\hline & & & $40-50$ & & $51-60$ & & $61-70$ & & $71-80$ & \\
\hline & $\beta \pm S E$ & $r$ & $\beta \pm S E$ & $r$ & $\beta \pm S E$ & $r$ & $\beta \pm S E$ & $r$ & $\beta \pm S E$ & $r$ \\
\hline $\mathrm{T}(\mathrm{nmol} / \mathrm{l})$ & $0 \cdot 25 \pm 0 \cdot 02$ & $0 \cdot 68^{\star * *}$ & $0 \cdot 26 \pm 0 \cdot 03$ & $0 \cdot 64^{* * \star}$ & $0 \cdot 26 \pm 0 \cdot 03$ & $0 \cdot 66^{\star * *}$ & $0 \cdot 26 \pm 0 \cdot 03$ & $0 \cdot 69^{* * *}$ & $0 \cdot 23 \pm 0 \cdot 03$ & $0 \cdot 66^{* * *}$ \\
\hline Non-SHBG-T $(\mathrm{nmol} / \mathrm{l})$ & $0 \cdot 03 \pm 0 \cdot 01$ & $0 \cdot 15^{\star}$ & $0 \cdot 02 \pm 0 \cdot 02$ & $0 \cdot 10 \mathrm{NS}$ & $0 \cdot 04 \pm 0 \cdot 02$ & $0 \cdot 20^{*}$ & $0 \cdot 04 \pm 0 \cdot 02$ & $0 \cdot 24^{*}$ & $0.02 \pm 0.01$ & $0 \cdot 15 \mathrm{NS}$ \\
\hline
\end{tabular}

${ }^{* * *} P<0 \cdot 001,{ }^{*} P<0 \cdot 05$, NS, not significant. All associations have been adjusted for age and BMI.

Fig. 2 SHBG vs. total testosterone (open symbols) and non-SHBG-T (closed symbols) in four age groups of adult men.
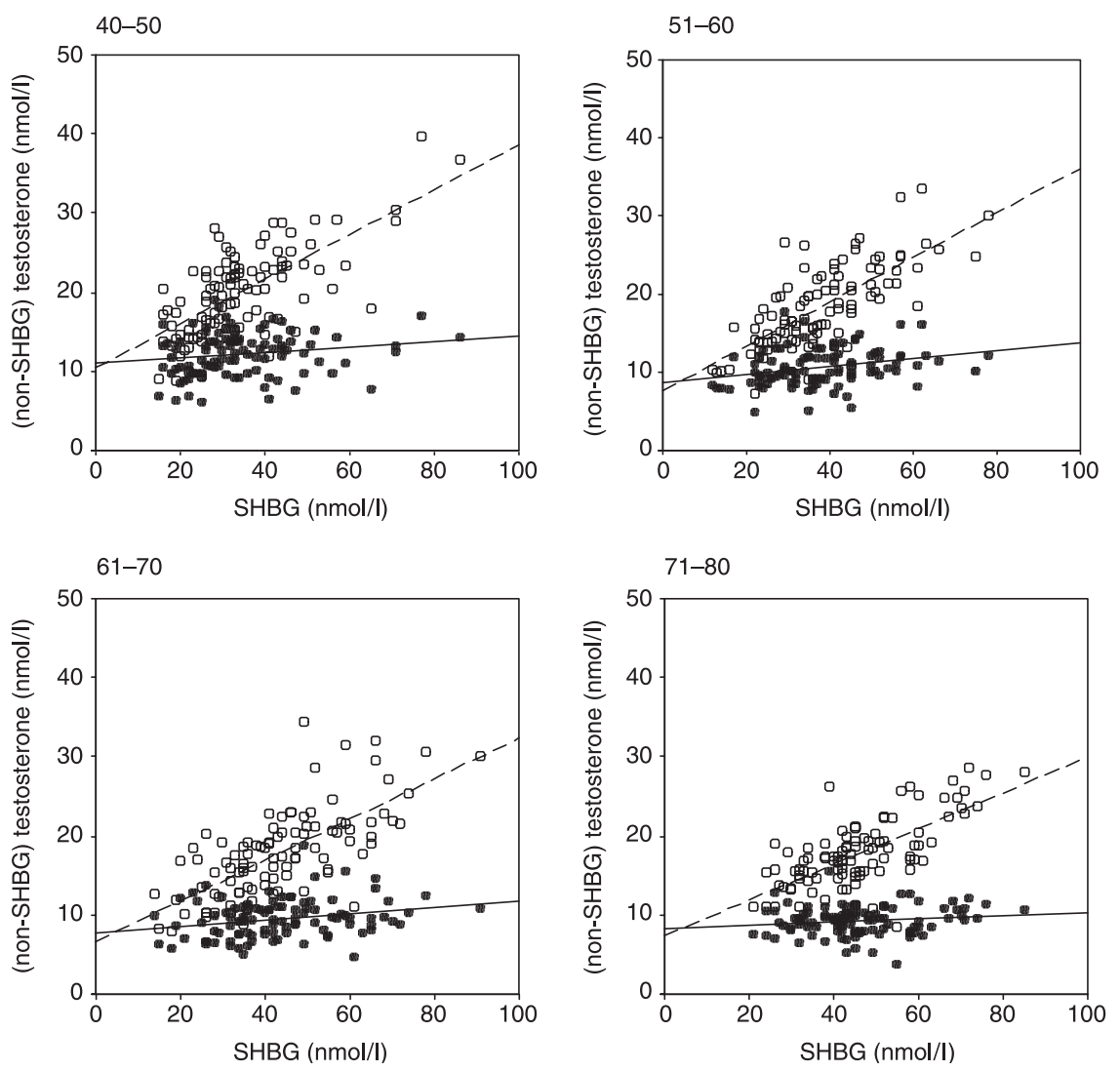

might also affect production rates of testosterone. It can be hypothesized that the SHBG-induced decreased bioavailability of testosterone will result in increased production of LH and subsequently testosterone until a new equilibrium is reached. SHBG decreases the metabolic clearance rate of testosterone in men and women., ${ }^{2,3}$ Higher levels of SHBG will thus be associated with higher levels of testosterone if the testosterone production rate does not change. However, in pre- and postmenopausal women, in whom testosterone is not directly involved in the regulation of the HPG axis, plasma testosterone levels were shown to be negatively correlated with SHBG levels, ${ }^{16}$ which argues against an important effect of SHBG on testosterone levels by effects on testosterone clearance alone. These observations support the conclusion that non-SHBG-T and not total testosterone is driving HPG axis activity in men.

The fact that in male neonates SHBG is not negatively associated with non-SHBG-T suggests that during the first months of life the
HPG axis is functional and sensitive to androgen feedback. This is supported by the observation that castration of neonatal monkeys results in elevated LH levels comparable to those found in castrated adults. ${ }^{17}$ In fact, androgen sensitivity appears to be a prerequisite for neonatal HPG axis activity as the postnatal hormone surge is absent in infants with androgen insensitivity. ${ }^{18}$ The absent relation between SHBG and non-SHBG-T in our subjects implies that the observed testosterone levels are not just the result of an unleashed HPG axis but are restrained by the feedback inhibition of non-SHBG-T. This is supported by the observation that $\mathrm{LH}$ and/or testosterone secretion in neonates can be further increased when stimulated with GnRH or human chorionic gonadotropin. ${ }^{19}$ Although the HPG axis is operative in neonates, circulating (non-SHBG) T levels are much lower than in adults. It appears that the higher SHBG levels found in neonates are not responsible for the relatively low levels of non-SHBG-T. A different setpoint of the HPG axis in neonates might thus be postulated. 
Our study confirms the well-known increase of SHBG and slight decrease of total testosterone with increasing age in adult men. ${ }^{12}$ This age related increase of SHBG is generally believed to be an important factor in the pronounced decline of non-SHBG-T in ageing men. However, in our cross-sectional analysis of different age groups we found that even in the oldest age group higher levels of SHBG are not associated with lower levels of non-SHBG-T. Therefore, the agerelated increase of SHBG does not appear to be responsible for the age-related decline of non-SHBG-T.

Several studies indicate that with age the sensitivity to the negative feedback action of sex hormones increases. ${ }^{20-22}$ Also, in our adult men, there seems to be a change of the setpoint of the HPG axis to testosterone feedback with ageing. The reason for this increased feedback sensitivity remains unknown. There are no arguments to believe that the expression of androgen action is stronger with age; the extent of SHBG suppression by androgens is similar in young and aged men. ${ }^{21}$

Ageing appears to be associated with combined hypothalamic and gonadal defects. However, the pituitary of elderly men responds adequately to stimulation by exogenous $\mathrm{LHRH}^{23}$ but the Leydig cell responsiveness to $\mathrm{LH}$ is decreased. ${ }^{24}$ With ageing, alterations in hypothalamic GnRH secretion are noted; decreased LH burst amplitude, increased LH burst frequency and a disordered LH burst pattern. Androgen deprivation reveals impaired GnRH secretory reserve in older men compared to young men. ${ }^{25}$ Our study shows that these defects of ageing do not cause an inadequate adaptation of testosterone concentrations to variations in SHBG levels.

In neonates, it appears that the HPG axis is even more sensitive to androgen feedback than the HPG axis of elderly men. The mechanism behind this is unknown. The response of the HPG axis to varying SHBG levels shows that the level of non-SHBG-T in newborns and adults is not solely a result of randomly combined SHBG and testosterone levels but the product of the HPG axis responding to negative and positive feedback signals. The precise nature and impact of these factors remain to be determined.

Our study has certain limitations. The adult volunteers for this study were all living independently. Especially in the highest age groups this might have led to a health selection bias.

The levels of testosterone in the neonates were low in comparison to adult men. Because commercial testosterone assays are designed to measure reliably in the adult range it is well known that they loose accuracy in the lower ranges. ${ }^{26}$ For several reasons we believe that our conclusions drawn from the neonatal data are justified. The method used in our study for the determination of testosterone showed the closest correlation with values determined by liquid chromatography-tandem mass spectrometry. ${ }^{26}$ The intra- and interassay coefficients of variation were below $9 \%$ for the concentration range measured in the samples and the mean testosterone level in our study was similar to levels reported by others. ${ }^{10}$ The studied population was large. Moreover, this study was not designed to make statements about individual hormone levels but to analyse relations between parameters in a group of individuals. The finding of a highly significant association between levels of total testosterone and SHBG in both neonates and adult men underlines the validity of our estimates; if the estimates for the testosterone concentration in neonates were highly inaccurate such a relation would not have been found.
The approach used in this study allows us to make statements about the setpoint of the HPG axis of a group of men but cannot be translated to individuals. For instance, it is well known that all kinds of diseases are associated with an impaired function of the HPG axis. ${ }^{27}$ When the HPG axis is compromised not only by age but also by other factors the capability to adapt to testosterone binding by SHBG might be lost. In those cases, SHBG may become a contributor to low non-SHBG-T levels in men.

The cross-sectional nature of this study inherently does not allow us to make definitive causative statements about the observed correlations between SHBG and sex hormones.

In conclusion, the results of our study show that in male neonates and in healthy adult men levels of SHBG, if at all, barely contribute to variations in non-SHBG-T.

\section{References}

1 Dunn, J.F., Nisula, B.C. \& Rodbard, D. (1981) Transport of steroid hormones: binding of 21 endogenous steroids to both testosteronebinding globulin and corticosteroid-binding globulin in human plasma. Journal of Clinical Endocrinology and Metabolism, 53, 58-68.

2 Vermeulen, A. \& Ando, S. (1979) Metabolic clearance rate and interconversion of androgens and the influence of the free androgen fraction. Journal of Clinical Endocrinology and Metabolism, 48, 320-326.

3 Saez, J.M., Forest, M.G., Morera, A.M. \& Bertrand, J. (1972) Metabolic clearance rate and blood production rate of testosterone and dihydrotestosterone in normal subjects, during pregnancy, and in hyperthyroidism. Journal of Clinical Investigation, 51, 1226-1234.

4 Mendel, C.M. (1989) The free hormone hypothesis: a physiologically based mathematical model. Endocrine Reviews, 10, 232-274.

5 Manni, A., Pardridge, W.M., Cefalu, W., Nisula, B.C., Bardin, C.W., Santner, S.J. \& Santen, R.J. (1985) Bioavailability of albumin-bound testosterone. Journal of Clinical Endocrinology and Metabolism, 61, 705-710.

6 Vermeulen, A., Verdonck, L. \& Kaufman, J.M. (1999) A critical evaluation of simple methods for the estimation of free testosterone in serum. Journal of Clinical Endocrinology and Metabolism, 84, 36663672 .

7 Longcope, C., Feldman, H.A., McKinlay, J.B. \& Araujo, A.B. (2000) Diet and sex hormone-binding globulin. Journal of Clinical Endocrinology and Metabolism, 85, 293-296.

8 Vermeulen, A., Kaufman, J.M. \& Giagulli, V.A. (1996) Influence of some biological indexes on sex hormone-binding globulin and androgen levels in aging or obese males. Journal of Clinical Endocrinology and Metabolism, 81, 1821-1826.

9 Mann, D.R. \& Fraser, H.M. (1996) The neonatal period: a critical interval in male primate development. Journal of Endocrinology, 149, 191-197.

10 Andersson, A.M., Toppari, J., Haavisto, A.M., Petersen, J.H., Simell, T., Simell, O. \& Skakkebaek, N.E. (1998) Longitudinal reproductive hormone profiles in infants: peak of inhibin B levels in infant boys exceeds levels in adult men. Journal of Clinical Endocrinology and Metabolism, 83, 675-681.

11 Belgorosky, A. \& Rivarola, M.A. (1986) Progressive decrease in serum sex hormone-binding globulin from infancy to late prepuberty in boys. Journal of Clinical Endocrinology and Metabolism, 63, $510-512$.

12 Harman, S.M., Metter, E.J., Tobin, J.D., Pearson, J. \& Blackman, M.R. (2001) Longitudinal effects of aging on serum total and free testosterone 
levels in healthy men. Baltimore Longitudinal Study of Aging. Journal of Clinical Endocrinology and Metabolism, 86, 724-731.

13 Sodergard, R., Backstrom, T., Shanbhag, V. \& Carstensen, H. (1982) Calculation of free and bound fractions of testosterone and estradiol$17 \beta$ to human plasma proteins at body temperature. Journal of Steroid Biochemistry, 16, 801-810.

14 de Ronde, W., van der Schouw, Y.T., Muller, M., Grobbee, D.E., Gooren, L.J., Pols, H.A. \& de Jong, F.H. (2005) Associations of sexhormone-binding globulin (SHBG) with non-SHBG-bound levels of testosterone and estradiol in independently living men. Journal of Clinical Endocrinology and Metabolism, 90, 157-162.

15 Muller, M., den Tonkelaar, I., Thijssen, J.H., Grobbee, D.E. \& van der Schouw, Y.T. (2003) Endogenous sex hormones in men aged 40-80 years. European Journal of Endocrinology, 149, 583-589.

16 Pasquali, R., Vicennati, V., Bertazzo, D., Casimirri, F., Pascal, G., Tortelli, O. \& Labate, A.M. (1997) Determinants of sex hormonebinding globulin blood concentrations in premenopausal and postmenopausal women with different estrogen status. Virgilio Menopause Health Group. Metabolism, 46, 5-9.

17 Plant, T.M. (1980) The effects of neonatal orchidectomy on the developmental pattern of gonadotropin secretion in the male rhesus monkey (Macaca mulatta). Endocrinology, 106, 1451-1454.

18 Bouvattier, C., Carel, J.C., Lecointre, C., David, A., Sultan, C., Bertrand, A.M., Morel, Y. \& Chaussain, J.L. (2002) Postnatal changes of T, LH, and FSH in 46,XY infants with mutations in the AR gene. Journal of Clinical Endocrinology and Metabolism, 87, 29-32.

19 Dunkel, L., Perheentupa, J., Tapanainen, J., Leinonen, P. \& Vihko, R. (1984) Responsiveness of the pituitary-testicular axis to gonadotropin-releasing hormone and chorionic gonadotropin during the first week of life. Pediatric Research, 18, 1085-1087.

20 Deslypere, J.P., Kaufman, J.M., Vermeulen, T., Vogelaers, D., Vandalem, J.L. \& Vermeulen, A. (1987) Influence of age on pulsatile luteinizing hormone release and responsiveness of the gonadotrophs to sex hormone feedback in men. Journal of Clinical Endocrinology and Metabolism, 64, 68-73.

21 Winters, S.J., Sherins, R.J. \& Troen, P. (1984) The gonadotropinsuppressive activity of androgen is increased in elderly men. Metabolism, 33, 1052-1059.

22 Winters, S.J. \& Atkinson, L. (1997) Serum LH concentrations in hypogonadal men during transdermal testosterone replacement through scrotal skin: further evidence that ageing enhances testosterone negative feedback. The Testoderm Study Group. Clinical Endocrinology, 47, 317-322.

23 Kaufman, J.M., Giri, M., Deslypere, J.M., Thomas, G. \& Vermeulen, A. (1991) Influence of age on the responsiveness of the gonadotrophs to luteinizing hormone-releasing hormone in males. Journal of Clinical Endocrinology and Metabolism, 72, 1255-1260.

24 Mulligan, T., Iranmanesh, A. \& Veldhuis, J.D. (2001) Pulsatile iv infusion of recombinant human LH in leuprolide-suppressed men unmasks impoverished Leydig-cell secretory responsiveness to midphysiological LH drive in the aging male. Journal of Clinical Endocrinology and Metabolism, 86, 5547-5553.

25 Veldhuis, J.D., Zwart, A., Mulligan, T. \& Iranmanesh, A. (2001) Muting of androgen negative feedback unveils impoverished gonadotropinreleasing hormone/luteinizing hormone secretory reactivity in healthy older men. Journal of Clinical Endocrinology and Metabolism, 86, 529-535.

26 Wang, C., Catlin, D.H., Demers, L.M., Starcevic, B. \& Swerdloff, R.S. (2004) Measurement of total serum testosterone in adult men: comparison of current laboratory methods versus liquid chromatography-tandem mass spectrometry. Journal of Clinical Endocrinology and Metabolism, 89, 534-543.

27 Baker, H.W. (1998) Reproductive effects of nontesticular illness. Endocrinology and Metabolism Clinics of North America, 27, 831-850. 